\title{
WINSURE: A NEW WINDOWS INTERFACE TO THE SURE PROGRAM
}

\author{
Ricky W. Butler, NASA Langley Research Center, Hampton, Va
}

\begin{abstract}
WinSURE is new interface to the SemiMarkov Range Evaluator (SURE) program, a reliability analysis program used for calculating upper and lower bounds on the operational and death state probabilities for a large class of semiMarkov models. The SURE program was developed in the late 1980s for the Unix environment and has been distributed free-ofcharge for over a decade. The WinSURE program is a port of the SURE program to the Windows 98 operating system, providing the same functionality as the original program, but with a Windows-based graphical user interface. The program provides a rapid computational capability for semi-Markov models useful in describing the fault-handling behavior of fault-tolerant computer systems. The only modeling restriction imposed by the program is that the nonexponential recovery transitions must be fast in comparison to the mission time- a desirable attribute of all fault-tolerant reconfigurable systems. The WinSURE reliability analysis method utilizes a fast bounding theorem based on means and variances that enables the calculation of upper and lower bounds on system reliability. The calculated bounds are close enough (usually within 5 percent of each other) for use in reliability studies of ultra-reliable computer systems. The SURE bounding theorems provide algebraic solutions to the underlying semi-Markov models and are consequently computationally efficient even for large and complex systems. The WinSURE user can specify a parameter to vary over a range of values and obtain an automatic sensitivity analysis.
\end{abstract}

The WinSURE program provides some new features not available in the original SURE program. The user is able to plot the results of the last run via a simple button push. The program accomplishes this by sending the results to the GNUPLOT program (a free program) which generates the plot. The WinSURE program also provides a capability to graphically display the input model. This is accomplished through use of another free program called VCG. The WinSURE user presses a button and the program automatically generates the appropriate input for VCG and executes it.

This paper will present an overview of the functionality of the WinSURE program, describe the graphical user interface, and illustrate the use of the program on some simple example problems.

\section{Introduction}

The WinSURE program is a flexible, userfriendly reliability analysis tool. It is a Windows 98 version of the SURE program developed in the early 1980's at NASA Langley Research Center. The program provides a rapid computational capability for semi-Markov models useful in describing the fault-handling behavior of faulttolerant computer systems. The only modeling restriction imposed by the program is that the nonexponential recovery transitions must be fast in comparison to the mission time - a desirable attribute of all fault-tolerant systems. The WinSURE reliability analysis method utilizes a fast bounding theorem based on means and variances. This bounding theorem enables the calculation of upper and lower bounds on system reliability. The upper and lower bounds are typically within about 5 percent of each other. Since the computation method is extremely fast, large state spaces are not a problem. The program provides mechanisms for model pruning that greatly increases the solution efficiency for large models. The operational state probabilities can optionally be calculated though the accuracy is less than the accuracy for the death states.

This paper describes the Windows interface to the WinSURE program. The reader is referred to the online documentation entitled "The SURE INPUT Language" for a detailed description of the model definition language and to [1] and [2] for a detailed description of the solution methods used. A tutorial/user's guide is available in [3]. 


\section{Basic Program Concept}

The user of the WinSURE program must first define a semi-Markov by enumerating all the transitions of the model using a simple language. This can be done inside the WinSURE program (See CREATE button), though the more powerful Emacs text editor is recommended. The states of the model are named with natural numbers. The semi-Markov input model is described by enumerating all the transitions between the states of the system. There are two different statements used to enter transitions---one for slow transitions and the other for fast. If a transition is slow, then the following type of statement is used:

$$
1,2=0.0001
$$

This defines a slow exponential transition from state 1 to state 2 with rate 0.0001 . The program does not require any particular units, e.g., hour ${ }^{-1}$ or $\mathrm{sec}^{-1}$. However, the user must use consistent units (i.e. if the mission time is specified in hours, then the rates should be hour ${ }^{-1}$ ). If the transition is fast, the following syntax is used:

$$
2,4=<1 \mathrm{E}-4,1 \mathrm{E}-6,1.0>\text {; }
$$

The numbers in the brackets correspond to the conditional mean, conditional standard deviation ${ }^{1}$, and transition probability of the fast transition, respectively.

\section{WinSURE By Way Of Example}

The semi-Markov model shown in figure 1 describes a quadraplex architecture that degrades to a triplex and then to a simplex in response to failures. The horizontal transitions represent fault arrivals. The coefficients of $\lambda$ represent the number of processors in the configuration. The vertical transitions represent recovery from a fault through removal of the faulty processor. Since the quadraplex system uses majority voting for fault masking, there is a race between the occurrence of fault $\# 2$ and removal of fault \#1. If fault \#2 wins the race, then the system fails (state 3 ).

${ }^{1}$ The mean and standard deviation are conditioned on the event that this transition succeeds over any other competing fast recoveries from the state. If there are no other fast recoveries from this state, the conditional mean and standard deviation are the same as the unconditionals.

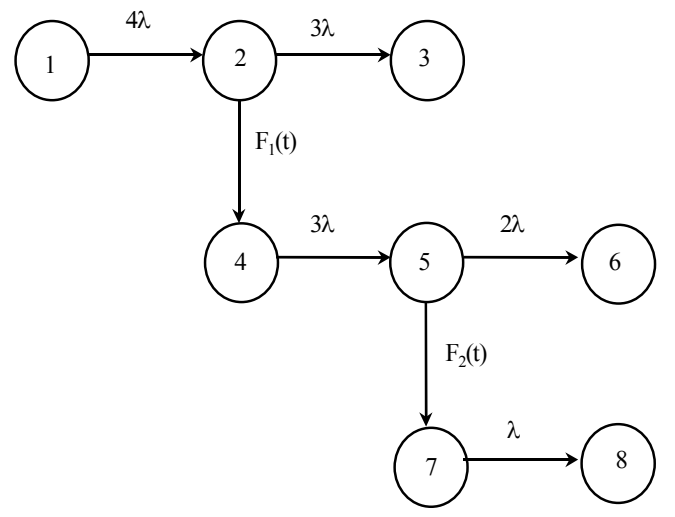

Figure 1. Semi-Markov Model of Quadraplex

This model is described by the following WinSURE input file

$$
\begin{aligned}
& \text { LAMBDA = 1E-4; } \\
& \text { MU1 = 2.7E-4; } \\
& \text { SIGMA1 = 1.3E-3; } \\
& \text { MU2 = 2.7E-4; } \\
& \text { SIGMA2 = 1.3E-3; } \\
& \text { 1,2 = 4*LAMBDA; } \\
& 2,3=3^{*} \text { LAMBDA; } \\
& 2,4=<\text { MU1,SIGMA1>; } \\
& \text { 4,5 = 3*LAMBDA; } \\
& 5,6=2 * \text { LAMBDA; } \\
& 5,7=<\text { MU2,SIGMA2>; } \\
& 7,8=\text { LAMBDA; }
\end{aligned}
$$

The first 5 statements equate values to identifiers (symbolic names). The identifier LAMBDA represents the processor failure rate. The identifiers MU1 and SIGMA1 are the mean and standard deviation of the time to remove a faulty processor. The identifiers MU2 and SIGMA2 are the mean and standard deviation of the time to degrade to a simplex. Conveniently, the only information WinSURE needs about the nonexponential recovery processes are the means and standard deviations. The final 7 statements define the transitions of the model. 


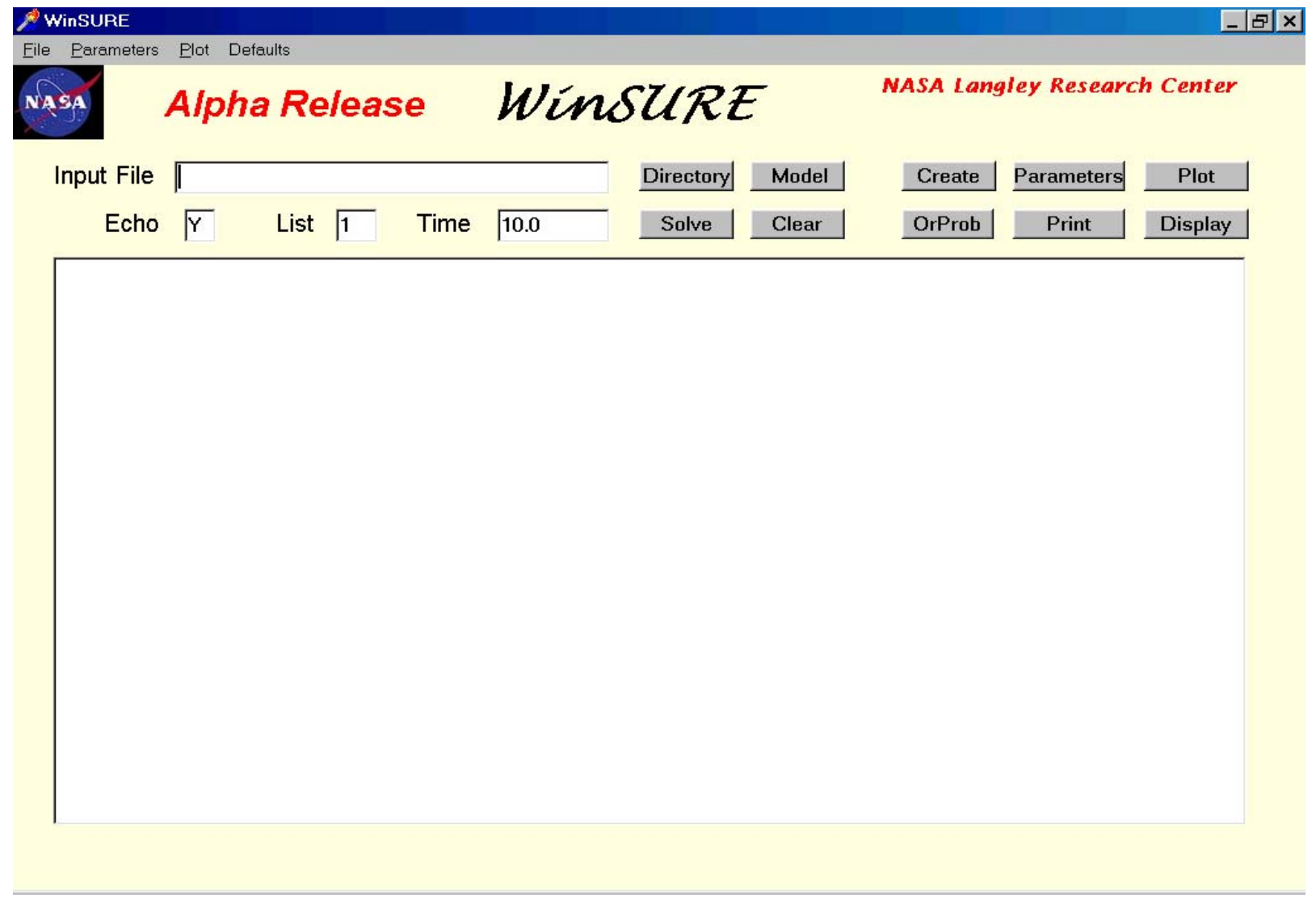

Figure 2. WinSURE Interface Window

If the transition is a fault-arrival (or slow), the transition is assumed to be exponentially distributed and only the exponential rate must be provided. For example, the last statement defines a transition from state 7 to state 8 with rate LAMBDA. If the transition is a recovery transition (or fast), the mean and standard deviation of the recovery time must be given. For example, the statement 2,4= $<$ MU1,SIGMA1 $>$ defines a transition from state 2 to state 4 with mean recovery time MU1 and standard deviation SIGMA1.

When the WinSURE program is started, the window shown in Figure 2 is displayed. The user then begins an interactive section by designating the working directory (i.e. the directory containing the model files) using the Directory button. The Model button is used to specify the file that contains the definition of the semi-Markov model to be solved. This text file can be created using a text editor such as Emacs or NotePad. Alternatively, the model can be created using the Create button; however, the editing features provided are minimal.

Once the model file has been selected its location appears in the box labeled input file. (If desired a user can directly enter the location of the file into this box.) The user then presses the Solve button to obtain the WinSURE analysis. The box labeled Time is used to specify the mission time, if it is not specified in the model file directly. The box labeled List is used to specify the level of detail of the output and the box labeled Echo determines whether the input model is listed in the window. Figure 3 illustrates the output that is received when the model (shown in Figure 1) is solved: 


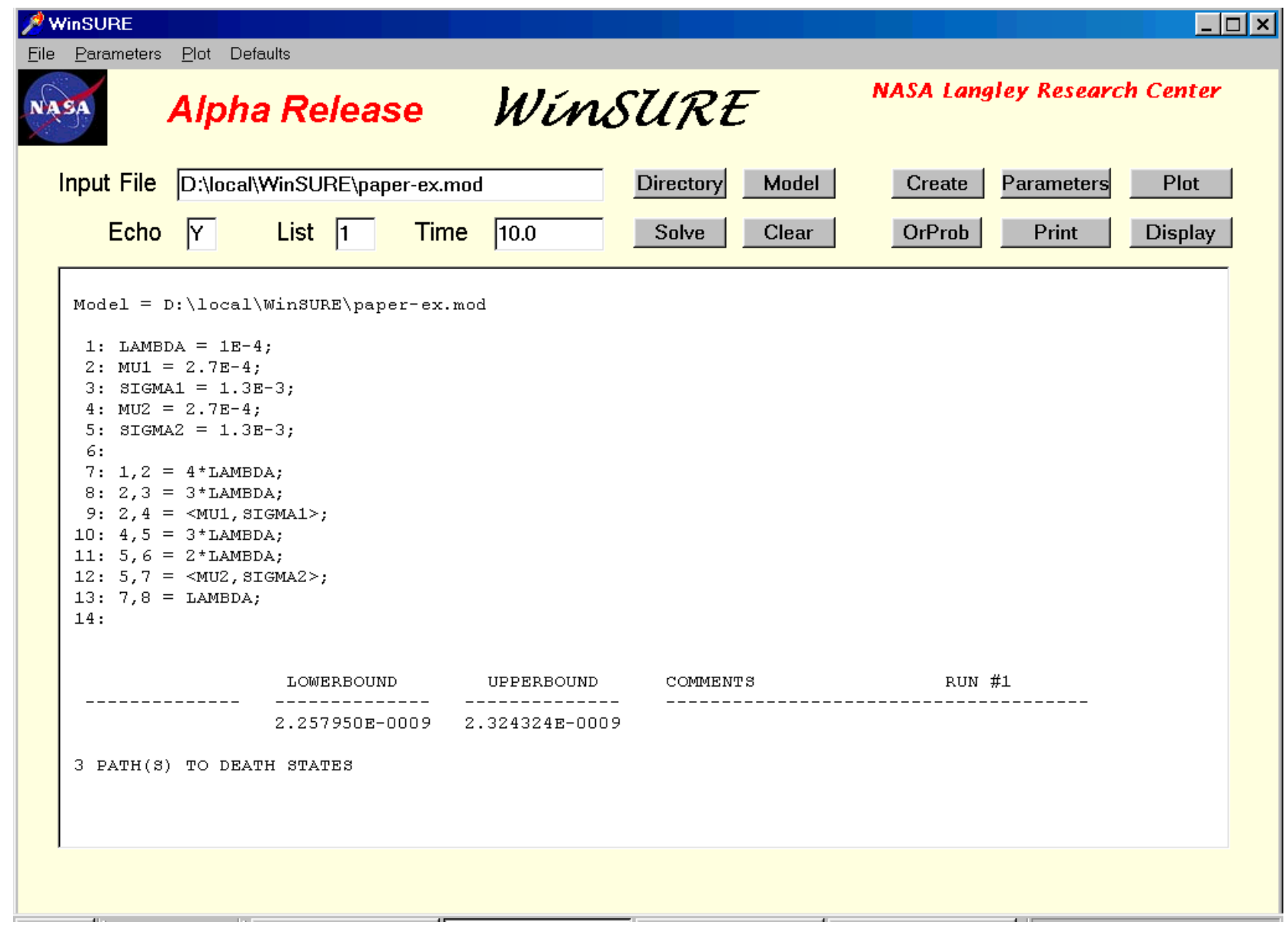

Figure 3. Output From Solution of TMR Model

Changing the box labeled List to 2, directs WinSURE to list the probability of entering each death state separately as shown in figure 4 .

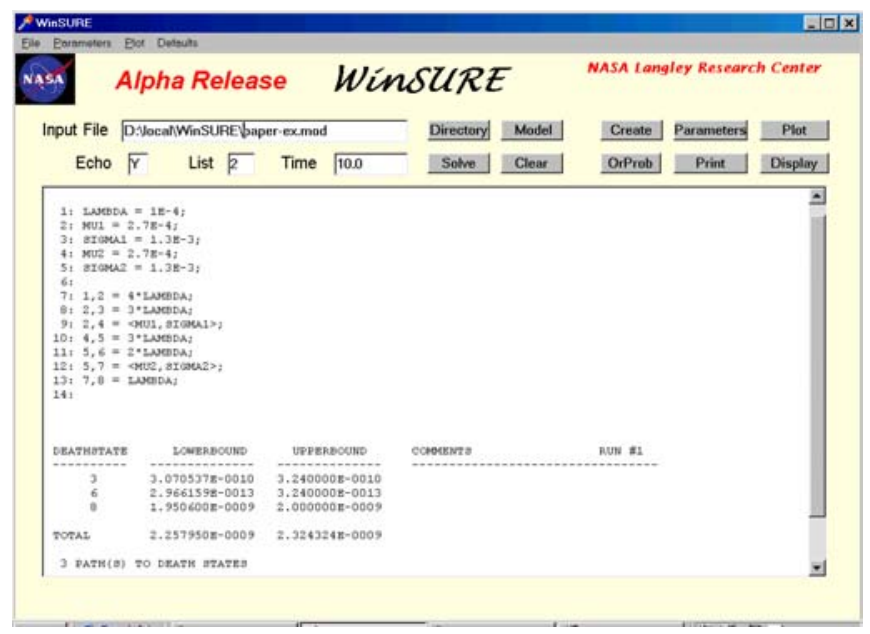

Figure 4. Output With List $=2$
If the LAMBDA statement is changed to the following:

$$
\text { LAMBDA = 1E-12 to* 1E-1 BY 10; }
$$

the WinSURE program solves the model repeatedly for the specified values of LAMBDA, namely

$$
\text { 1E-12, 1E-11, 1E-10, 1E-9, .. 1E-1. }
$$

The output from the model, using this definition of LAMBDA, is shown in figure 5. 


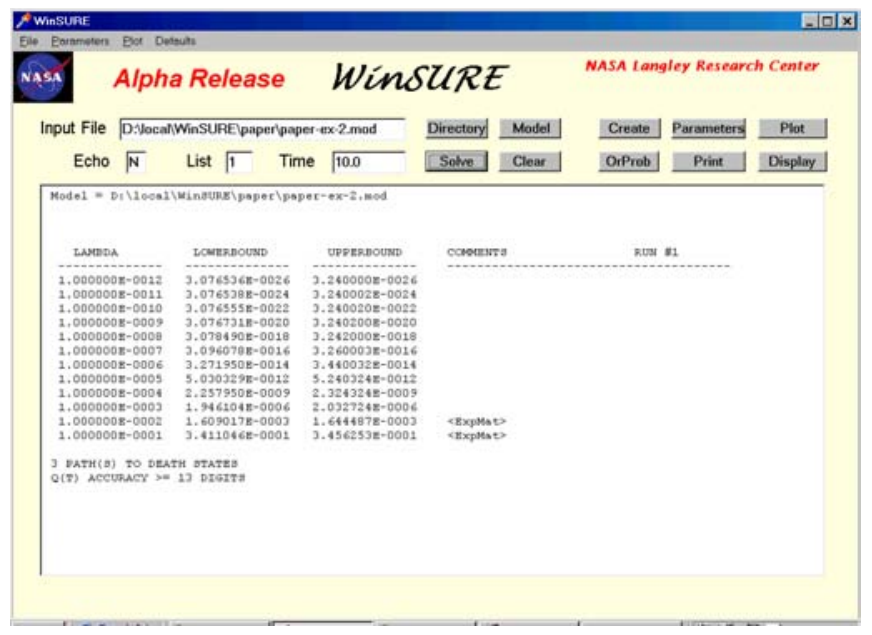

Figure 5. Output from Variable LAMBDA

Because the Echo Box was changed to "N", the model file was not listed.

If the user then presses the Plot button the results are plotted ${ }^{2}$ :

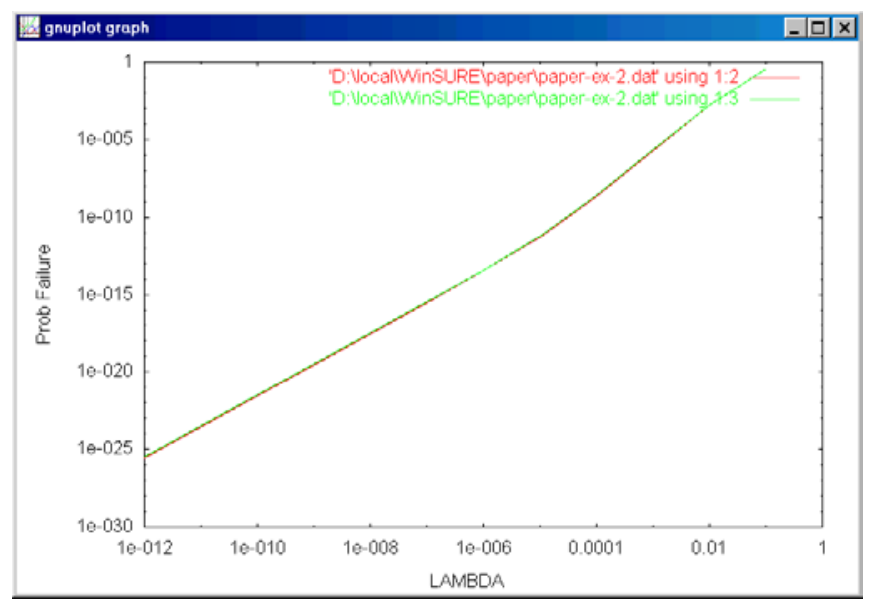

Figure 6. Plot Produced By WinSURE

Alternatively, you can start GNUPLOT and issue the GNUPLOT command load 'sure.plt' after pressing the Plot button or after issuing the Write Plot File command on the Plot menu.

${ }^{2}$ GNUPLOT must be installed and registered to open .plt files in order for this to work. The GNUPLOT software is copyrighted but freely distributed at http://www.cs.dartmouth.edu/gnuplot_info.html
The model file can be graphically displayed using the Display button ${ }^{3}$.

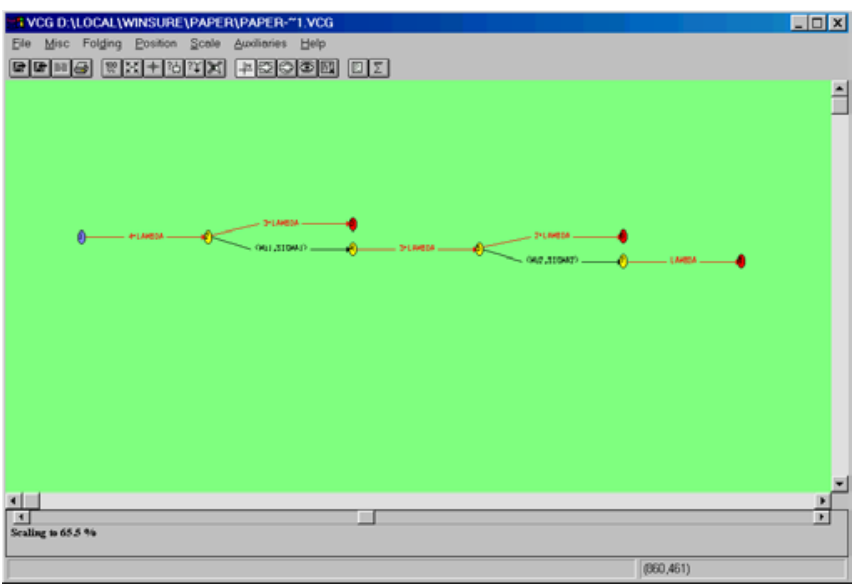

Figure 7. Graphical Display of Model

Alternatively, you can start VCG and load the .vcg file created when the DISPLAY button is pressed.

\section{The WinSURE Interface Details}

\section{Directory Button}

The Directory button is used to select the working directory (i.e. the directory where the model files are stored and the output files are written.)

\section{Model Button}

The Model button is used to select the model to be solved.

\section{Solve Button}

The Solve button is used to solve the selected model. The probability that the system enters any of the death states within the specified mission time is computed. The results are displayed in the window and optionally written to a .run file. The amount of detail is determined by the value of List.

${ }^{3}$ VCG must be installed and registered to open .vcg files in order for this to work The VCG software is available by anonymous ftp at ftp.cs.uni-sb.de (134.96.7.254) in the directory/pub/graphics/vcg. It is freely available under the GNU General Public License. 
This parameter can be set in the model file or through the List box:

$0=$ No output is sent to the window

$1=$ Only the total system failure probability is listed

$2=$ The probability bounds for each death state in the model are reported along with the totals

$3=$ The probability for the operational states are reported in addition to the death states

$4=$ Every path in the model is listed and its probability of traversal.

\section{Clear Button} Window.

The Clear button is used to clear the Output

\section{Create Button}

The Create button is used to create a new model. It opens up an edit dialog window (shown in figure 8) in which a new model file can be entered and saved:

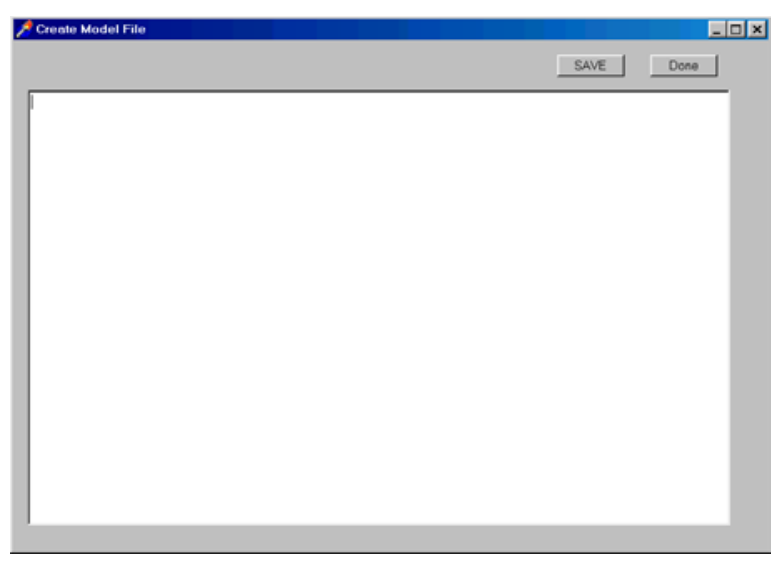

Figure 8. New Model Window

\section{Parameters Button}

The parameter button opens the window shown in figure 9.

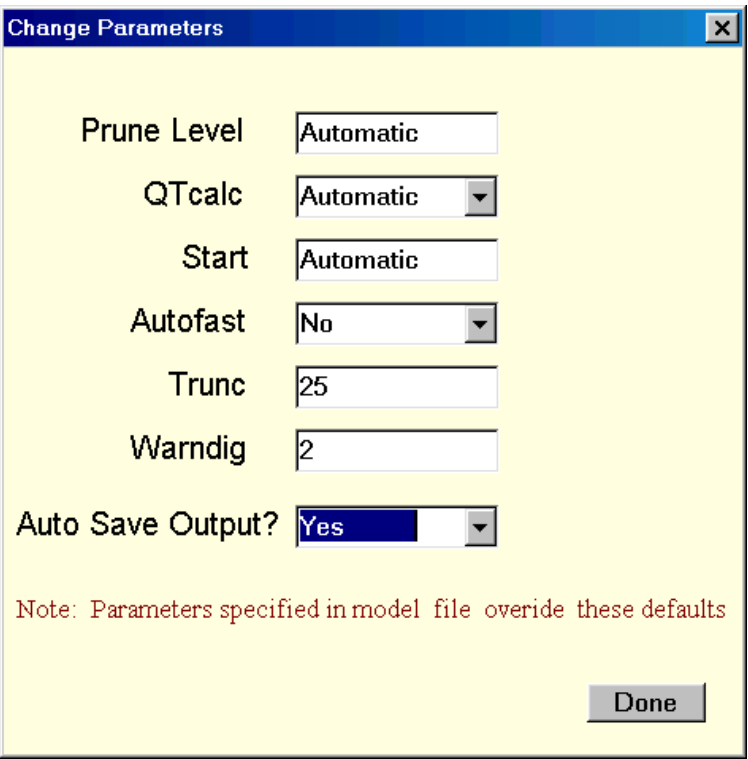

Figure 9. Change Parameters Window

Each of the edit boxes available in this window are described below:

Prune Level: The SURE program follows paths in the model until they reach a death state or the probability drops below the Prune level specified here. If this parameter is set to Automatic, the prune level is determined automatically. The error due to pruning is always added to the upper bound, so the results are always conservative.

QTcalc: $\quad 0=$ use algebraic method (FAST) to calculate $\mathrm{Q}(\mathrm{T})$

$1=$ use algebraic method (MORE ACCURATE) to calculate $\mathrm{Q}(\mathrm{T})$

Automatic $=$ Let WinSURE decide on a path-by-path basis.

Start: The Start constant can be used to specify the start state. If set to Automatic, the program will use the source state (i.e. the state with no transitions into it). If set to Automatic and there is no source state the first state entered will be the start state.

Autofast: If set to YES or 1, the program will accept fast exponential transitions without the FAST keyword. 
Trunc: This parameter sets the maximum number of times that WinSURE will unfold a fast loop. Note: models that contain fast loops, i.e. loops with only fast transitions, can cause the program to run forever unless this "safety value" is used. Fast loops generate an infinite sequence of paths which do not decrease in probability (as far as WinSURE's Upper Bound is concerned).

Warndig: Issue warning when the number of digits of accuracy in the results are less than this value.

Auto Save Output?: If set to YES, the output will be written to a file as well as to the window. The file name is the model name with a .run extension. This is especially useful for large output, which exceeds the capacity of the window.

\section{Plot Button}

The PLOT button calls GNUPLOT to plot results of the last solution.

\section{OrProb Button}

The OrProb button computes the probabilistic OR of all of the runs since the last Clear command.

\section{Print Button}

The Print button directs WinSURE to print contents of output window.

\section{Display Button}

The Display button directs WinSURE to use VCG to graphically display the model on the screen.

\section{File Menu}

The File menu provides a menu interface to (1) open and create models files, (2) print, save and clear the output window, (3) run a file list, and (4) exit. All of these functions except (3) can also be accomplished by using one of the buttons described above.

The Run File List item opens a new window:

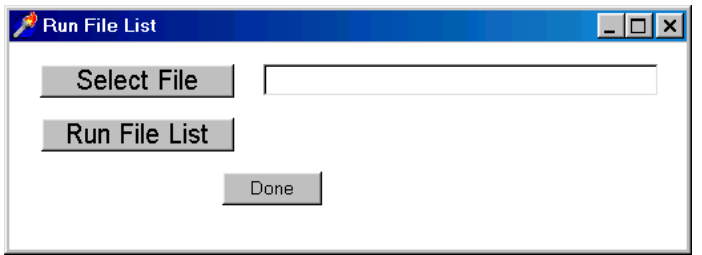

that allows the user to provide WinSURE with a file containing a list of model files to be solved. When the Run File List button is pressed, WinSURE solves all of the models listed in the specified file.

\section{Parameters Menu}

The Parameters menu opens the same parameters window that the Parameters button opens.

\section{Plot Menu}

The Plot menu has three items: (1) Plot Options, (2) Gnuplot Execute, and (3) Write Plot File.

The Plot Options item opens the following window:

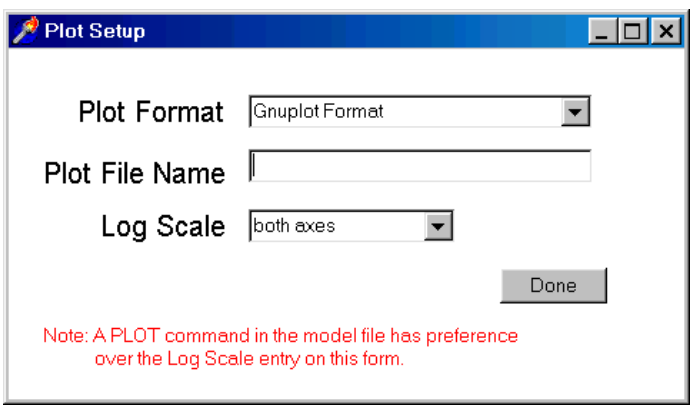

which allows the user to (a) select either GNUPLOT format or Matlab format, (b) change the name of the plot file, and (c) specify which axes should be logarithmically scaled.

The Gnuplot Execute item calls GNUPLOT to plot the results and the Write Plot File item just writes out a file that can be processed by GNUPLOT or Matlab subsequently ${ }^{4}$.

\footnotetext{
${ }^{4}$ The WinSURE program closes the plot output files as soon as the PLOT command completes, so that GNUPLOT can run immediately after the PLOT command in a separate $\mathrm{X}$ window.
} 


\section{Defaults Menu}

The Defaults menu allows the user to save the current values of parameters and options so that subsequent executions of WinSURE will be loaded with the desired values.

\section{Quick Review Of Model Definition Language}

The SURE user defines an input model in an text file. The basic idea is to enumerate all of the transitions between states using two types of statements. The first type is used to define a slow transition. The transition is completely specified by citing the source state (source), the destination state (dest), and the transition rate (rate) as follows:

source, dest $=$ rate;

The second type of transition defines a fast transition

$$
\text { source, dest }=<m u, \operatorname{sig}\{, \text { frac }\}>\text {; }
$$

where $m u$ is the conditional mean transition time, sig is the conditional standard deviation of the transition time, and frac is the transition probability ${ }^{5}$. The third parameter is optional when there is only one fast transition from a state. The states are named with integers up to an installation parameter MAXSTATE usually $1,000,000^{6}$. The other parameters (i.e. rate, $m u$, sig, and frac) can be defined using arithmetic expressions. The following are all valid SURE statements:

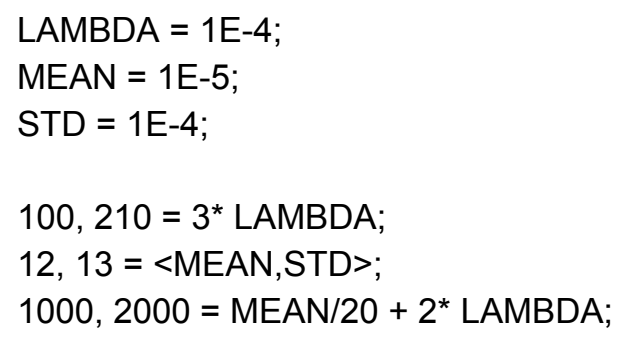

\footnotetext{
${ }^{5}$ The transition probability is the probability that this fast transition succeeds over any other fast transitions. The conditioning of the other parameters is on this transition succeeding. If there is only one fast transition from a state, the transition probability frac is 1 .

${ }^{6}$ This limit can only be changed by redefining the constant in the SURE program and recompiling the SURE source.
}

The input model may contain one parameter which is given a range of values. The SURE system will compute the system failure probability as a function of this variable. The following are examples:

$$
\begin{aligned}
& \text { LAMBDA = 1E-9 TO* } 1 \mathrm{E}-4 \text { BY } 10 ; \\
& C=0.5 \text { TO+ } 0.9 \text { BY 0.1; }
\end{aligned}
$$

The * after the TO keyword indicates that the range is geometric. The LAMBDA variable above is specified to be $1 \mathrm{E}-9,1 \mathrm{E}-8,1 \mathrm{E}-7,1 \mathrm{E}-6,1 \mathrm{E}-5$ and 1E-4. The + after the TO keyword indicates that the range is arithmetic. The variable $C$ above is specified to be $0.5,0.6,0.7,0.8$, and 0.9. Only one such variable may be defined.

For a more detailed definition of the input language the reader is referred to [3].

\section{Concluding Remarks}

The WinSURE program is an Semi-Markov model reliability analysis program that runs on the Windows operating system. It computes the death state and operational state probabilities for userinput models. Beta versions of the program are available upon request ${ }^{7}$.

\section{References}

[1] Butler, Ricky W., Allan L. White, 1988, SURE Reliability Analysis: Program and Mathematics. NASA Technical Paper 2764, Mar. 1988.

[2] Butler, Ricky W.: The SURE Approach to Reliability Analysis. IEEE Transactions on Reliability, vol. 41, no. 2, June 1992, pp. 210--218.

[3] Ricky W. Butler and Sally C. Johnson, Techniques for Modeling the Reliability of Fault-Tolerant Systems With the Markov State-Space Approach , NASA RP-1348, September 1995, pp. 130.

\footnotetext{
${ }^{7}$ Foreign release is more difficult but can be approved for applications that involve public safety.
} 